\title{
From dynamic to static and back: Riding the roller coaster of information-flow control research
}

\author{
Andrei Sabelfeld and Alejandro Russo \\ Dept. of Computer Science and Engineering, Chalmers University of Technology \\ 41296 Göteborg, Sweden, Fax: +46 317723663
}

\begin{abstract}
Historically, dynamic techniques are the pioneers of the area of information flow in the 70's. In their seminal work, Denning and Denning suggest a static alternative for information-flow analysis. Following this work, the 90's see the domination of static techniques for information flow. The common wisdom appears to be that dynamic approaches are not a good match for security since monitoring a single path misses public side effects that could have happened in other paths. Dynamic techniques for information flow are on the rise again, driven by the need for permissiveness in today's dynamic applications. But they still involve nontrivial static checks for leaks related to control flow.

This paper demonstrates that it is possible for a purely dynamic enforcement to be as secure as Denning-style static information-flow analysis, despite the common wisdom. We do have the trade-off that static techniques have benefits of reducing runtime overhead, and dynamic techniques have the benefits of permissiveness (this, for example, is of particular importance in dynamic applications, where freshly generated code is evaluated). But on the security side, we show for a simple imperative language that both Denning-style analysis and dynamic enforcement have the same assurance: termination-insensitive noninterference.
\end{abstract}

\section{Introduction}

Historically, dynamic techniques are the pioneers of the area of information flow in the 70's (e.g., [9]). They prevent explicit flows (as in public := secret) in program runs. They also address implicit [8] flows (as in if secret then public $:=1$ ) by enforcing a simple invariant of no public side effects in secret context, i.e., in the branches of conditionals and loops with secret guards. These techniques, however, come without soundness arguments.

In their seminal paper, Denning and Denning [8] suggest a static alternative for information-flow analysis. They argue that static analysis removes runtime overhead for security checks. This analysis prevents both explicit and implicit flows statically. The invariant of no public side effects in secret context is ensured by a syntactic check: no assignments to public variables are allowed in secret context. Denning and Denning do not discuss soundness, but Volpano et al. [27] show soundness by proving termination-insensitive noninterference, when they cast Denning and Denning's analysis as a security type system. Termination-insensitive noninterference guarantees that starting with two initial memories that agree on public data, two terminating runs of a 
program result in final memories that also agree on public data. Denning-style analysis is by now the core for the information-flow tools Jif [15], FlowCaml [22], and the SPARK Examiner [4, 7].

The 90's see the domination of static techniques for information flow [19]. The common wisdom appears to be that dynamic approaches are not a good match for security since monitoring a single path misses public side effects that could have happened in other paths.

For example, Myers and Liskov [14] discuss:

... static checking allows precise, fine-grained analysis of information flows, and can capture implicit flows properly, whereas dynamic label checks create information channels that must be controlled through additional static checking...

As can be seen from above (the emphasis is ours), it appears to be suggested that dynamic checking alone is insufficient for security.

Dynamic techniques for information flow are on the rise again $[25,13,21,12,24]$ driven by the need for permissiveness in today's dynamic applications. But they still involve nontrivial static checks for leaks related to control flow.

In this light, it might be surprising that it is possible for purely dynamic enforcement to be as secure as Denning-style static analysis. The key factor is termination. Program constructs introduce channels for information transfer (recall the explicit and implicit flows above that correspond to channels via assignments and branching). The termination channel is introduced by loops: by observing the termination of program while secret do skip, the attacker learns that secret was 0 . Denning-style static analyses are typically termination-insensitive. They ignore leaks via the termination behavior of programs. Thus, they satisfy termination-insensitive noninterference [27], as previously mentioned. Monitors supervise the execution of programs to guarantee security properties. Executed instructions are first analyzed by the monitor to determine if they are safe to run. In the presence of unsafe instructions, monitors can take several countermeasures: block the execution of programs or transform the unsafe instruction into a safe one. If the monitor can introduce nontermination by blocking the underlying program, this feature can be used for collapsing high-bandwidth information channels into low-bandwidth ones. Turning the high-bandwidth implicit-flow channel into the lowbandwidth termination channel is one example: blocking the execution at an attempt of a public assignment in secret context (note the similarities to the techniques from the 70 's!) is in fact sufficient for termination-insensitive security.

This paper demonstrates the above insight for a simple imperative language. We present a Denning-style static analysis in the form of a security type system by Volpano et al. [27] and a simple monitor. We show that a monitor is strictly more permissive than the type system, and both the type system and the monitor satisfy terminationinsensitive noninterference.

Sections 2-5 consider a batch-job model: programs run until completion before the produce a result (which is the final memory). Termination-insensitive noninterference [27] for batch-job programs simply ignores diverging runs. However, Section 6 generalizes our results to a language with output, a natural extension [1] of the type system by Volpano et al. [27] with output, and progress-insensitive noninterference [1], 


$$
\begin{aligned}
& \langle\text { skip, } m\rangle \stackrel{n o p}{\longrightarrow}\langle\text { stop }, m\rangle \\
& \frac{m(e)=v}{\langle x:=e, m\rangle \stackrel{a(x, e)}{\longrightarrow}\langle s t o p, m[x \mapsto v]\rangle} \\
& \frac{\left\langle c_{1}, m\right\rangle \stackrel{\alpha}{\longrightarrow}\left\langle\text { stop, } m^{\prime}\right\rangle}{\left\langle c_{1} ; c_{2}, m\right\rangle \stackrel{\alpha}{\longrightarrow}\left\langle c_{2}, m^{\prime}\right\rangle} \quad \frac{\left\langle c_{1}, m\right\rangle \stackrel{\alpha}{\longrightarrow}\left\langle c_{1}^{\prime}, m^{\prime}\right\rangle \quad c_{1}^{\prime} \neq \text { stop }}{\left\langle c_{1} ; c_{2}, m\right\rangle \stackrel{\alpha}{\longrightarrow}\left\langle c_{1}^{\prime} ; c_{2}, m^{\prime}\right\rangle} \\
& \frac{m(e) \neq 0}{\left\langle\text { if } e \text { then } c_{1} \text { else } c_{2}, m\right\rangle \stackrel{b(e)}{\longrightarrow}\left\langle c_{1} ; \text { end }, m\right\rangle} \quad \frac{m(e)=0}{\left\langle\text { if } e \text { then } c_{1} \text { else } c_{2}, m\right\rangle \stackrel{b(e)}{\longrightarrow}\left\langle c_{2} ; \text { end }, m\right\rangle} \\
& \frac{m(e) \neq 0}{\langle\text { while } e \text { do } c, m\rangle \stackrel{b(e)}{\longrightarrow}\langle c ; \text { end; while } e \text { do } c, m\rangle} \quad \frac{m(e)=0}{\langle\text { while } e \text { do } c, m\rangle \stackrel{b(e)}{\longrightarrow}\langle\text { end, } m\rangle} \\
& \langle e n d, m\rangle \stackrel{f}{\longrightarrow}\langle\text { stop, } m\rangle
\end{aligned}
$$

Fig. 1. Command semantics

a generalization of termination-insensitive noninterference to reason about programs with output, which does not ignore diverging runs, but ignores (the lack of) progress at each step.

\section{Semantics}

Figure 1 presents the semantics for a simple imperative language. Configurations have the form $\langle c, m\rangle$, where $c$ is a command and $m$ is a memory mapping variables to values. Semantic rules have the form $\langle c, m\rangle \stackrel{\alpha}{\longrightarrow}\left\langle c^{\prime}, m^{\prime}\right\rangle$, which corresponds to a small step between configurations. If a transition leads to a configuration with the special command stop and some memory $m$, then we say the execution terminates in $m$. Observe that there are no transitions triggered by stop. The special command end signifies exiting the scope of an if or a while. Observe that end is executed after the branches of those commands. Commands stop and end can be generated during execution of programs but they are not used in initial configurations, i.e., they are not accesible to programmers. For simplicity, we consider simple integer expressions in our language (i.e., constants, binary operations, and variables). The semantics for expressions is then standard and thus we omit it here. We note the result of evaluating expression $e$ under memory $m$ as $m(e)$. The semantics are decorated with events $\alpha$ for communicating program events to an execution monitor. Event nop signals that the program performs a skip. Event $a(x, e)$ records that the program assigns the value of $e$ in the current memory to variable $x$. Event $b(e)$ indicates that the program branches on expression $e$. Finally, event $f$ is generated when the structure block of a conditional or loop has finished evaluation.

Assume $c f g, c f g^{\prime}, \ldots$ range over command configurations and $c f g m, c f g m^{\prime}, \ldots$ range over monitor configurations. For this work, it is enough to think of monitor con- 


$$
\begin{array}{ccc}
p c \vdash \operatorname{skip} & \frac{\operatorname{lev}(e) \sqsubseteq \Gamma(x) \quad p c \sqsubseteq \Gamma(x)}{p c \vdash x:=e} & \frac{p c \vdash c_{1} \quad p c \vdash c_{2}}{p c \vdash c_{1} ; c_{2}} \\
\frac{\operatorname{lev}(e) \sqcup p c \vdash c_{1} \quad l e v(e) \sqcup p c \vdash c_{2}}{p c \vdash \text { if } e \text { then } c_{1} \text { else } c_{2}} & \frac{l e v(e) \sqcup p c \vdash c}{p c \vdash \text { while } e \text { do } c}
\end{array}
$$

Fig. 2. Typing rules

$s t \stackrel{n o p}{\longrightarrow} s t \quad \frac{l e v(e) \sqsubseteq \Gamma(x) \quad l e v(s t) \sqsubseteq \Gamma(x)}{s t \stackrel{a(x, e)}{\longrightarrow} s t} \quad s t \stackrel{b(e)}{\longrightarrow} l e v(e): s t \quad h d: s t \stackrel{f}{\longrightarrow} s t$

Fig. 3. Monitoring rules

figurations as simple stacks of security levels (see below). The semantics are parametric in the monitor $\mu$, which is assumed to be described by transitions between monitor configurations in the form $c f g m \stackrel{\alpha}{\longrightarrow} \mu f g m^{\prime}$. The rule for monitored execution is:

$$
\frac{c f g \stackrel{\alpha}{\longrightarrow} c f g^{\prime} \quad c f g m \stackrel{\alpha}{\longrightarrow} \mu f g m^{\prime}}{\left\langle\left. c f g\right|_{\mu} c f g m\right\rangle \longrightarrow\left\langle\left. c f g^{\prime}\right|_{\mu} c f g m^{\prime}\right\rangle}
$$

The simplest example of a monitor is an all-accepting monitor $\mu_{0}$, which is defined by $\epsilon \stackrel{\alpha}{\longrightarrow} \mu_{0} \epsilon$, where $\epsilon$ is its only state (the empty stack). This monitor indeed accepts all events $\alpha$ in the underlying program.

\section{Type system}

Figure 2 displays a Denning-style static analysis in the form of a security type system by Volpano et al. [27]. Typing environment $\Gamma$ maps variables to security levels in a security lattice. For simplicity, we assume a security lattice with two levels $L$ and $H$ for low (public) and high (secret) security, where $L \sqsubset H$. Function lev $(e)$ returns $H$ if there is a high variable in $e$ and otherwise returns $L$. Typing judgment for commands has the form $p c \vdash c$, where $p c$ is a security level known as the program counter that keeps track of the context. Explicit flows (as in $l:=h$ ) are prevented by the typing rule for assignment that disallows assignments of high expressions to low variables. Implicit flows (as in if $h$ then $l:=1$ else $l:=0$ ) are prevented by the $p c$ mechanism. It demands that when branching on a high expression, the branches must be typed under high $p c$, which prevents assignments to low variables in the branches.

\section{Monitor}

Figure 3 presents monitor $\mu_{1}$ (we omit the subscript $\mu_{1}$ in the transition rules for clarity). The monitor either accepts an event generated by the program or blocks it by getting stuck. The monitor configuration $s t$ is a stack of security levels, intended to keep track 
of the current security context: the security levels of the guards of conditionals and loops whose body the computation currently visits. This is a dynamic version of the $p c$ from the previous section. Event nop (that originates from a skip) is always accepted without changes in the monitor state. Event $a(x, e)$ (that originates from an assignment) is accepted without changes in the monitor state but with two conditions: (i) that the security level of expression $e$ is no greater than the security level of variable $x$ and (ii) that the highest security level in the context stack (denoted lev $(s t)$ for a stack $s t$ ) is no greater than the security level of variable $x$. The former prevents explicit flows of the form $l:=h$, whereas the latter prevents implicit flows of the form if $h$ then $l:=$ 1 else $l:=0$, where depending on the high guard, the execution of the program leads to different low events.

Events $b(e)$ result in pushing the security level of $e$ onto the stack of the monitor. This is a part of implicit-flow prevention: runs of program if $h$ then $l:=1$ else $l:=0$ are blocked before performing an assignment $l$ because the level of the stack is high when reaching the execution of the assignment. The stack structure avoids overrestrictive enforcement. For example, runs of program (if $h$ then $h:=1$ else $h:=0) ; l:=$ 1 are allowed. This is because by the time the assignment to $l$ is reached, the execution has left the high context: the high security level has been popped from the stack in response to event $f$, which the program generates on exiting the if.

We have seen that runs of programs like if $h$ then $l:=1$ else $l:=0$ are rejected by the monitor. But what about a program like if $h$ then $l:=1$ else skip, a common example for illustrating that dynamic information-flow enforcement is delicate? If $h$ is nonzero, the monitor blocks the execution. However, if $h$ is 0 , the program proceeds normally. Are we accepting an insecure program? It turns out that the slight difference between unmonitored and monitored runs (blocking in case $h$ is nonzero) is sufficient for termination-insensitive security. In effect, the monitor prevents implicit flows by collapsing the implicit-flow channel into the termination channel; it does not introduce any more bandwidth than what the termination channel already permits. Indeed, implicit flows in unmonitored runs can be magnified by a loop so that secrets can be leaked bit-by-bit in linear time in the size of the secret. On the other hand, implicit flows in monitored runs cannot be magnified because execution is blocked whenever it attempts entering a branch with a public side effect. For example, one implication for uniformly-distributed secrets is that they cannot be leaked on the termination channel in polynomial time [1].

\section{Results}

This section presents the formal results. We assume $\mu_{0}$ is the monitor that accepts all program events, and $\mu_{1}$ is the monitor from Section 4. First, we show that the monitor $\mu_{1}$ is strictly more permissive than the type system. If a program is typable, then all of its runs are not modified by the monitor.

Theorem 1. If $p c \vdash c$ and $\left\langle\left.\langle c, m\rangle\right|_{\mu_{0}} \epsilon\right\rangle \longrightarrow^{*}\left\langle\left.\left\langle c^{\prime}, m^{\prime}\right\rangle\right|_{\mu_{0}} \epsilon\right\rangle$, then $\left\langle\left.\langle c, m\rangle\right|_{\mu_{1}} \epsilon\right\rangle \longrightarrow^{*}$ $\left\langle\left.\left\langle c^{\prime}, m^{\prime}\right\rangle\right|_{\mu_{1}} s t^{\prime}\right\rangle$.

Proof. We prove a generalization of the theorem (see the Appendix). Intuitively, the theorem holds because (i) the requirements for assignments in the type system and the 


$$
\frac{m(e)=v}{\langle\operatorname{output}(e), m\rangle \stackrel{o(e)}{\longrightarrow}_{v}\langle\text { stop, } m\rangle} \quad \frac{l e v(e) \sqcup p c \sqsubseteq L}{p c \vdash \operatorname{output}(e)} \quad \frac{l e v(e) \sqcup s t \sqsubseteq L}{s t \stackrel{o(e)}{\longrightarrow} s t}
$$

Fig. 4. Semantics, typing, and monitoring rules for outputs

monitor $\mu_{1}$ are essentially the same; and (ii) there is a tight relation between the join operations for $p c$ and pushing security levels on the stack $s t$.

Further, there are programs (e.g., if $l>l$ then $l:=h$ else skip) whose runs are always accepted by the monitor, but which are rejected by the type system. Hence, the monitor is strictly more permissive than the type system.

We now show that both the type system and monitor enforce the same security condition: termination-insensitive noninterference [27]. Two memories $m_{1}$ and $m_{2}$ are lowequal (written $m_{1}={ }_{L} m_{2}$ ) if they agree on the low variables. Termination-insensitive noninterference demands that starting with two low-equal initial memories, two terminating runs of a typable program result in low-equal final memories.

Theorem 2. If $p c \vdash c$, then for all $m_{1}$ and $m_{2}$, where $m_{1}={ }_{L} m_{2}$, whenever we have $\left\langle\left.\left\langle c, m_{1}\right\rangle\right|_{\mu_{0}} \epsilon\right\rangle \longrightarrow^{*}\left\langle\left.\left\langle s t o p, m_{1}^{\prime}\right\rangle\right|_{\mu_{0}} \epsilon\right\rangle$ and $\left\langle\left.\left\langle c, m_{2}\right\rangle\right|_{\mu_{0}} \epsilon\right\rangle \longrightarrow^{*}\left\langle\left.\left\langle s t o p, m_{2}^{\prime}\right\rangle\right|_{\mu_{0}} \epsilon\right\rangle$, then $m_{1}^{\prime}={ }_{L} m_{2}^{\prime}$.

Proof. By adjusting the soundness proof by Volpano et al. [27] (see the Appendix).

Termination-insensitive noninterference also holds for the runs monitored by the monitor from Section 4:

Theorem 3. For all $m_{1}$ and $m_{2}$, where $m_{1}={ }_{L} m_{2}$, whenever c contains no end commands and $\left\langle\left.\left\langle c, m_{1}\right\rangle\right|_{\mu_{1}} \epsilon\right\rangle \longrightarrow^{*}\left\langle\left.\left\langle s t o p, m_{1}^{\prime}\right\rangle\right|_{\mu_{1}} s t_{1}^{\prime}\right\rangle$ and $\left\langle\left.\left\langle c, m_{2}\right\rangle\right|_{\mu_{1}} \epsilon\right\rangle \longrightarrow^{*}$ $\left\langle\left.\left\langle\right.\right.$ stop, $\left.m_{2}^{\prime}\right\rangle\right|_{\mu_{1}}$ st $\left.t_{2}^{\prime}\right\rangle$, then $m_{1}^{\prime}={ }_{L} m_{2}^{\prime}$.

Proof. By induction on $\longrightarrow^{*}$. The details can be found in the Appendix.

\section{Incorporating output into the language}

This section introduces outputs to the language. For simplicity, we only consider public outputs. The semantics, typing, and monitoring rules for outputs are described in Figure 4. Command output $(e)$ outputs the value of expression $e$ on a public channel. Semantically, configurations might now trigger externally observable events with an additional label $(v)$ indicating an output value. Public outputs can be considered as special assignments to low variables. In this light, the typing and monitor rules (adapted from [1] and [2], respectively) for this command are similar to the ones applied when modifying low variables. Event $o(e)$ conveys information that expression $e$ is output by the program. Monitored configurations need to be adapted to synchronize with output events. Formally, a monitor transition $\left\langle\left. c f g\right|_{\mu} c f g m\right\rangle \longrightarrow_{\gamma}\left\langle\left. c f g^{\prime}\right|_{\mu} c f g m^{\prime}\right\rangle$ is possible if 
the program and monitor transitions $c f g \stackrel{\alpha}{\longrightarrow} c f g^{\prime}$ and $c f g m \stackrel{\alpha}{\longrightarrow} c f g m^{\prime}$ are also possible. Event $\alpha$ can be $o(e)$ or any of the events described in Section 4. Event $\gamma$ stands for an externally observable event: it can be an output $(v)$ or an empty event $(\epsilon)$.

We present the adaptation of Theorems 1-3 for a language with outputs (proved in an accompanying technical report [20]). The next theorem looks the same as Theorem 1 except for the presence of a vector of output events $(\vec{\gamma})$.

Theorem 4. If $p c \vdash c$ and $\left\langle\left.\langle c, m\rangle\right|_{\mu_{0}} \epsilon\right\rangle \longrightarrow \vec{\gamma}^{*}\left\langle\left.\left\langle c^{\prime}, m^{\prime}\right\rangle\right|_{\mu_{0}} \epsilon\right\rangle$, then there exists st' such that $\left\langle\left.\langle c, m\rangle\right|_{\mu_{1}} \epsilon\right\rangle \longrightarrow \vec{\gamma}^{*}\left\langle\left.\left\langle c^{\prime}, m^{\prime}\right\rangle\right|_{\mu_{1}} s t^{\prime}\right\rangle$.

As before, there are programs (e.g., if $l>l$ then $l:=h$ else skip) whose runs are always accepted by the monitor, but which are rejected by the type system. Hence, the monitor for the extended language is strictly more permissive than the extended type system.

As explained in Section 1, Sections 2-5 consider a batch-job model: programs run until completion before the produce a result (which is the final memory). Terminationinsensitive noninterference [27] for batch-job programs simply ignores diverging runs. This condition is not appropriate for reasoning about programs with output since a program that outputs a secret and then diverges would be secure[3,1]. Thus, the security condition guarantee for the extended type system establishes progress-insensitive noninterference [1], a generalization of termination-insensitive noninterference to reason about programs with output, which does not ignore diverging runs, but ignores (the lack of) progress at each step. We show that given two low-equivalent initial memories, and the two sequences of outputs generated by monitored executions in these memories, then either the sequences are the same or one of them is a prefix of the other, in which case the execution that generates the shorter sequence produces no further public output events. Formally:

Theorem 5. If $p c \vdash c$, then for all $m_{1}$ and $m_{2}$, where $m_{1}={ }_{L} m_{2}$, whenever we have $\left\langle\left\langle c, m_{1}\right\rangle \mid \mu_{0} \epsilon\right\rangle \longrightarrow{\overrightarrow{\gamma_{1}}}^{*}\left\langle\left\langle s t o p, m_{1}^{\prime}\right\rangle \mid \mu_{\mu_{0}} s t_{1}^{\prime}\right\rangle$, then there exists $c^{\prime}, m_{2}^{\prime}, s t_{2}^{\prime}, \overrightarrow{\gamma_{2}}$ such that $\left\langle\left.\left\langle c, m_{2}\right\rangle\right|_{\mu_{0}} \epsilon\right\rangle \longrightarrow{\overrightarrow{\gamma_{2}}}^{*}\left\langle\left.\left\langle c^{\prime}, m_{2}^{\prime}\right\rangle\right|_{\mu_{0}} s t_{2}^{\prime}\right\rangle$ where $\left|\overrightarrow{\gamma_{2}}\right| \leq\left|\overrightarrow{\gamma_{1}}\right|$, and

a) If $\left|\overrightarrow{\gamma_{2}}\right|=\left|\overrightarrow{\gamma_{1}}\right|$, then $\overrightarrow{\gamma_{1}}=\overrightarrow{\gamma_{2}}$.

b) If $\left|\overrightarrow{\gamma_{2}}\right|<\left|\overrightarrow{\gamma_{1}}\right|$, then prefix $\left(\overrightarrow{\gamma_{2}}, \overrightarrow{\gamma_{1}}\right)$ holds and $\left\langle\left\langle c^{\prime}, m_{2}^{\prime}\right\rangle \mid \mu_{0} s t^{\prime}\right\rangle \Rightarrow_{H}$.

The number of events in $\vec{\gamma}$ is denoted by $|\vec{\gamma}|$. We also define predicate prefix $(\vec{x}, \vec{y})$ to hold when list $\vec{x}$ is a prefix of list $\vec{y}$. We write $\left\langle\langle c, m\rangle \mid{ }_{\mu} c f g m\right\rangle \Rightarrow_{H}$ to denote a monitored execution that does not produce any public output. Generalized terminationinsensitive noninterference also holds for the extended monitor. More precisely, we have the following theorem.

Theorem 6. For all $m_{1}$ and $m_{2}$, where $m_{1}=_{L} m_{2}$, whenever $c$ contains no end commands and $\left\langle\left\langle c, m_{1}\right\rangle \mid \mu_{\mu_{1}} \epsilon\right\rangle \longrightarrow{\overrightarrow{\gamma_{1}}}^{*}\left\langle\left.\left\langle s t o p, m_{1}^{\prime}\right\rangle\right|_{\mu_{1}}\right.$ st $\left.t_{1}^{\prime}\right\rangle$ then there exists $c^{\prime}, m_{2}^{\prime}, s_{2}^{\prime}, \overrightarrow{\gamma_{2}}$ such that $\left\langle\left.\left\langle c, m_{2}\right\rangle\right|_{\mu_{1}} \epsilon\right\rangle \longrightarrow{\overrightarrow{\gamma_{2}}}^{*}\left\langle\left\langle c^{\prime}, m_{2}^{\prime}\right\rangle||_{\mu_{1}}\right.$ st $\left.{ }_{2}^{\prime}\right\rangle$ where $\left|\overrightarrow{\gamma_{2}}\right| \leq\left|\overrightarrow{\gamma_{1}}\right|$, and

a) If $\left|\overrightarrow{\gamma_{2}}\right|=\left|\overrightarrow{\gamma_{1}}\right|$, then $\overrightarrow{\gamma_{1}}=\overrightarrow{\gamma_{2}}$.

b) If $\left|\overrightarrow{\gamma_{2}}\right|<\left|\overrightarrow{\gamma_{1}}\right|$, then prefix $\left(\overrightarrow{\gamma_{2}}, \overrightarrow{\gamma_{1}}\right)$ holds and $\left\langle\left\langle c^{\prime}, m_{2}^{\prime}\right\rangle \mid \mu_{\mu_{1}} s t_{2}^{\prime}\right\rangle \Rightarrow_{H}$. 


\section{Discussion}

On joint points The monitor critically relies on the joint-point information for each branching point (in conditionals in loops). This allows the monitor to discover that the execution has left a secret context, and relax restrictions on assignment to public variables. When branching, the command end is inserted at the joint point by the semantics in Figure 1. At the time of execution, end communicates information that a joint point has been reached to the monitor.

In a more complex language, we would expect the interpreter/compiler to extract the information about joint points from the scopes in the program text. This might be natural in a structured language. We remark, however, that in a low-level languages, or in a language with breaks and continues, this might require a separate static analysis.

On flow sensitivity Another point to emphasize is regarding flow sensitivity, i.e., possibility for variables to store values of different sensitivity (low and high) over the course of computation. Although it might be against the intuition, if we consider a flow-sensitive type system [11], then it is actually impossible to have a purely dynamic sound mechanism that is more precise than the type system. We give the formal details in a separate paper [17], and illustrate the issue with an example (similar examples have been discussed in the literature [24, 6]). In the following program, assume secret is a high variable containing a boolean secret (either 0 or 1 ):

$$
\begin{aligned}
& \text { public }:=1 ; \text { temp }:=0 ; \\
& \text { if secret then temp }:=1 ; \\
& \text { if temp } \neq 1 \text { then public }:=0
\end{aligned}
$$

Imagine a simple purely dynamic monitor that keeps track of security levels of variables and updates them on each assignment in the following way. The monitor sets the level of the assigned variable to high in case there is a high variable on the right-hand side of the assignment or in case the assignment appears inside of a high context. The level of the variable is set to low in case there are no high variables in the right-hand side of the assignment and the assignment does not appear in high context. Otherwise, the monitor does not update the the level of the assigned variable. This is a straightforward extension of the monitor from Section 4 with flow sensitivity.

This monitor labels public and temp as low after the first two assignments because the variables receive low information (constants). If secret is nonzero, variable temp becomes high after the first conditional. In this case the guard in the second conditional is false, and so the then branch with the assignment public $:=0$ is not taken. Therefore, the monitor allows this execution. If secret is zero, then temp is not relabeled to high, and so the second if is also allowed by the monitor even though the then branch is taken: because it branches on an expression that does not involve high variables. As a result, the value of secret is leaked into public, which is missed by the monitor.

This illustrates that flow sensitivity introduces a channel that poses a challenge for purely dynamic enforcement. 


\section{Related work}

Fenton [9] presents a monitor that takes into account program structure. It keeps track of the security context stack, similarly to ours. However, Fenton does not discuss soundness with respect to noninterference-like properties. Volpano [25] considers a monitor that only checks explicit flows. Implicit flows are allowed, and therefore the monitor does not enforce noninterference. Boudol [5] revisits Fenton's work and observes that the intended security policy "no security error" corresponds to a safety property, which is stronger than noninterference. Boudol shows how to enforce this safety property with a type system.

Mechanisms by Venkatakrishnan et al. [23], Le Guernic et al. [13, 12], and Shroff et al. [21] combine dynamic and static checks. They have a number of attractive features, for example, the mechanism by Le Guernic et al. [13, 12] is flow-sensitive: security levels of variables may change during the program execution. We take a deeper look at the impact of flow sensitivity on the trade off between static and dynamic informationflow enforcement in a separate paper [17] (cf. discussion in Section 7).

Tracking information flow in web applications is becoming increasingly important (e.g., recent highlights are a server-side mechanism by Huang et al. [10] and a clientside mechanism for JavaScript by Vogt et al. [24], although they do not discuss soundness). Dynamism of web applications puts higher demands on the permissiveness of the security mechanism: hence the importance of dynamic analysis.

Yet, all the mechanisms from the above two paragraphs involve nontrivial static analysis for side effects in conditionals and loops, whereas our proof-of-concept monitor is purely dynamic.

The monitor presented here is at core of (i) the termination-insensitive part of the enforcement of information-release (or declassification) policies by Askarov and Sabelfeld [2] for a language with dynamic code evaluation and communication and (ii) the monitor by Russo and Sabelfeld [18] to secure programs with timeout instructions.

\section{Concluding remarks}

When it comes to information-flow tracking, static techniques have benefits of reducing runtime overhead, and dynamic techniques have the benefits of permissiveness (this, for example, is of particular importance in dynamic applications, where freshly generated code is evaluated). But on the security side, we have demonstrated that both Denning-style analysis and dynamic enforcement have the same guarantees: termination-insensitive noninterference. Another way to interpret the result is that neither Denning-style analysis nor termination-insensitive noninterference itself offer strong guarantees (as also hinted in previous findings [1]).

However, when termination-sensitive noninterference is desired, the absence of side effects of traces not taken is hard to ensure dynamically.

But which policy should be the one of choice, termination-insensitive noninterference or termination-sensitive noninterference? Termination-sensitive noninterference is attractive, but rather difficult to guarantee. Typically, strong restrictions (such as no loops with secret guards [26]) are enforced. Program errors exacerbate the problem. 
Even in languages like Agda [16], where it is impossible to write nonterminating programs, it is possible to write programs that terminate abnormally: for example, with a stack overflow. Generally, abnormal termination due to resource exhaustion, is a channel for leaks that can be hard to counter.

As mentioned earlier, the information-flow tools Jif [15], FlowCaml [22], and the SPARK Examiner [4, 7] avoid these problems by targeting termination-insensitive noninterference. The price is that the attacker may leak secrets by brute-force attacks via the termination channel. But there is formal assurance that these are the only possible attacks. Askarov et al. [1] show that if a program satisfies termination-insensitive noninterference, then the attacker may not learn the secret in polynomial running time in the size of the secret; and, for uniformly-distributed secrets, the probability of guessing the secret in polynomial running time is negligible.

Acknowledgments Thanks are due to Gurvan Le Guernic and Rustan Leino for the interesting discussions. This work was funded by the Swedish research agencies SSF and VR.

\section{References}

[1] A. Askarov and S. Hunt and A. Sabelfeld and D. Sands. Termination-insensitive noninterference leaks more than just a bit. In Proc. European Symp. on Research in Computer Security, volume 5283 of LNCS, pages 333-348. Springer-Verlag, Oct. 2008.

[2] A. Askarov and A. Sabelfeld. Tight enforcement of information-release policies for dynamic languages. In Proc. IEEE Computer Security Foundations Symposium, July 2009.

[3] A. Banerjee, D. Naumann, and S. Rosenberg. Expressive declassification policies and modular static enforcement. In Proc. IEEE Symp. on Security and Privacy, May 2008.

[4] J. Barnes and J. Barnes. High Integrity Software: The SPARK Approach to Safety and Security. Addison-Wesley Longman Publishing Co., Inc. Boston, MA, USA, 2003.

[5] G. Boudol. Secure information flow as a safety property. In Formal Aspects in Security and Trust, Third International Workshop (FAST'08), LNCS, pages 20-34. Springer-Verlag, Mar. 2009.

[6] L. Cavallaro, P. Saxena, and R. Sekar. On the limits of information flow techniques for malware analysis and containment. In Proc. Detection of Intrusions and Malware \& Vulnerability Assessment (DIMVA), July 2008.

[7] R. Chapman and A. Hilton. Enforcing security and safety models with an information flow analysis tool. ACM SIGAda Ada Letters, 24(4):39-46, 2004.

[8] D. E. Denning and P. J. Denning. Certification of programs for secure information flow. Comm. of the ACM, 20(7):504-513, July 1977.

[9] J. S. Fenton. Memoryless subsystems. Computing J., 17(2):143-147, May 1974.

[10] Y.-W. Huang, F. Yu, C. Hang, C.-H. Tsai, D.-T. Lee, and S.-Y. Kuo. Securing web application code by static analysis and runtime protection. In Proc. International Conference on World Wide Web, pages 40-52, May 2004.

[11] S. Hunt and D. Sands. On flow-sensitive security types. In Proc. ACM Symp. on Principles of Programming Languages, pages 79-90, 2006.

[12] G. Le Guernic. Automaton-based confidentiality monitoring of concurrent programs. In Proc. IEEE Computer Security Foundations Symposium, pages 218-232, July 2007.

[13] G. Le Guernic, A. Banerjee, T. Jensen, and D. Schmidt. Automata-based confidentiality monitoring. In Proc. Asian Computing Science Conference (ASIAN'06), volume 4435 of LNCS. Springer-Verlag, 2006. 
[14] A. C. Myers and B. Liskov. Complete, safe information flow with decentralized labels. In Proc. IEEE Symp. on Security and Privacy, pages 186-197, May 1998.

[15] A. C. Myers, L. Zheng, S. Zdancewic, S. Chong, and N. Nystrom. Jif: Java information flow. Software release. Located at http://www.cs.cornell.edu/jif, July 2001.

[16] U. Norell. Towards a practical programming language based on dependent type theory. $\mathrm{PhD}$ thesis, Department of Computer Science and Engineering, Chalmers University of Technology, SE-412 96 Göteborg, Sweden, September 2007.

[17] A. Russo and A. Sabelfeld. Dynamic vs. static flow-sensitive security analysis, Apr. 2009. Draft.

[18] A. Russo and A. Sabelfeld. Securing timeout instructions in web applications. In Proc. IEEE Computer Security Foundations Symposium, July 2009.

[19] A. Sabelfeld and A. C. Myers. Language-based information-flow security. IEEE J. Selected Areas in Communications, 21(1):5-19, Jan. 2003.

[20] A. Sabelfeld and A. Russo. From dynamic to static and back: Riding the roller coaster of information-flow control research. full version. http://www.cse.chalmers.se/ russo/, 2009.

[21] P. Shroff, S. Smith, and M. Thober. Dynamic dependency monitoring to secure information flow. In Proc. IEEE Computer Security Foundations Symposium, pages 203-217, July 2007.

[22] V. Simonet. The Flow Caml system. Software release. Located at http://cristal.inria.fr/ simonet/soft/flowcaml, July 2003.

[23] V. N. Venkatakrishnan, W. Xu, D. C. DuVarney, and R. Sekar. Provably correct runtime enforcement of non-interference properties. In Proc. International Conference on Information and Communications Security, pages 332-351. Springer-Verlag, Dec. 2006.

[24] P. Vogt, F. Nentwich, N. Jovanovic, E. Kirda, C. Kruegel, and G. Vigna. Cross-site scripting prevention with dynamic data tainting and static analysis. In Proc. Network and Distributed System Security Symposium, Feb. 2007.

[25] D. Volpano. Safety versus secrecy. In Proc. Symp. on Static Analysis, volume 1694 of LNCS, pages 303-311. Springer-Verlag, Sept. 1999.

[26] D. Volpano and G. Smith. Eliminating covert flows with minimum typings. Proc. IEEE Computer Security Foundations Workshop, pages 156-168, June 1997.

[27] D. Volpano, G. Smith, and C. Irvine. A sound type system for secure flow analysis. $J$. Computer Security, 4(3):167-187, 1996.

\section{A Appendix}

Before proving the theorems described in body of the paper, we need to introduce some auxiliary lemmas. We describe the most important ones here. We start by showing lemmas related to sequential composition of monitored executions.

Lemma 1. If $\left\langle\left.\langle c, m\rangle\right|_{\mu} s t\right\rangle \longrightarrow^{*}\left\langle\left.\left\langle s t o p, m^{\prime}\right\rangle\right|_{\mu} s t^{\prime}\right\rangle$, then st $=s t^{\prime}$, where $\mu \in$ $\left\{\mu_{0}, \mu_{1}\right\}$.

Lemma 2. Given that stop; $c^{\prime}$ denotes $c^{\prime}$, if $\left\langle\left.\left\langle c_{1}, m\right\rangle\right|_{\mu}\right.$ st $\rangle \longrightarrow^{*}\left\langle\left.\left\langle c^{\prime}, m^{\prime}\right\rangle\right|_{\mu}\right.$ st $\left.t^{\prime}\right\rangle$, then $\left\langle\left.\left\langle c_{1} ; c_{2}, m\right\rangle\right|_{\mu} s t\right\rangle \longrightarrow^{*}\left\langle\left.\left\langle c^{\prime} ; c_{2}, m^{\prime}\right\rangle\right|_{\mu}\right.$ st' $\rangle$, where $\mu \in\left\{\mu_{0}, \mu_{1}\right\}$.

Lemma 3. If $\left\langle\left.\left\langle c_{1} ; c_{2}, m\right\rangle\right|_{\mu} s t\right\rangle \longrightarrow^{*}\left\langle\left.\left\langle c^{\prime}, m^{\prime}\right\rangle\right|_{\mu}\right.$ st' $\rangle$ and $c_{1}$ contains no end instructions, then there exists $c^{*}, m^{\prime \prime}$, and $s t^{*}$ such that $c^{\prime}=c^{*} ; c_{2}$ and $\left\langle\left.\left\langle c_{1}, m\right\rangle\right|_{\mu} s t\right\rangle \longrightarrow^{*}$ $\left\langle\left.\left\langle c^{*}, m^{\prime}\right\rangle\right|_{\mu} s t^{*}\right\rangle ;$ or $\left\langle\left.\left\langle c_{1}, m\right\rangle\right|_{\mu} s t\right\rangle \longrightarrow^{*}\left\langle\left.\left\langle s t o p, m^{\prime \prime}\right\rangle\right|_{\mu} s t\right\rangle$ and $\left\langle\left.\left\langle c_{2}, m^{\prime \prime}\right\rangle\right|_{\mu} s t\right\rangle \longrightarrow^{*}$ $\left\langle\left.\left\langle c^{\prime}, m^{\prime}\right\rangle\right|_{\mu} s t^{\prime}\right\rangle$, where $\mu \in\left\{\mu_{0}, \mu_{1}\right\}$. 
These lemmas can be proved by a simple induction on $\longrightarrow^{*}$. Before proving Theorem 1, we prove a generalization of it described in the following lemma.

Lemma 4. If $p c \vdash c,\left\langle\left.\langle c, m\rangle\right|_{\mu_{0}} \epsilon\right\rangle \longrightarrow^{*}\left\langle\left.\left\langle c^{\prime}, m^{\prime}\right\rangle\right|_{\mu_{0}} \epsilon\right\rangle$, then it holds $\forall$ lev(st $) \sqsubseteq$ $p c \cdot \exists \operatorname{lev}\left(s t^{\prime}\right) \cdot\left\langle\left.\langle c, m\rangle\right|_{\mu_{1}} s t\right\rangle \longrightarrow{ }^{*}\left\langle\left.\left\langle c^{\prime}, m^{\prime}\right\rangle\right|_{\mu_{1}} s t^{\prime}\right\rangle$.

Proof. By induction on $\longrightarrow^{*}$ and the number of sequential instructions in $c$. We only show the most interesting cases.

$x:=e$ ) Given a $s t$ such that $l e v(s t) \sqsubseteq p c$, we need to prove that exists $s t^{\prime}$ such that $l e v\left(s t^{\prime}\right)$ and $\left\langle\left.\langle x:=e, m\rangle\right|_{\mu_{1}} s t\right\rangle \longrightarrow\left\langle\left\langle s t o p, m^{\prime}\right\rangle \mid \mu_{1} s t^{\prime}\right\rangle$. Let's take $s t^{\prime}=$ st. Then, the transition under $\mu_{1}$ is possible provided that lev $(e) \sqsubseteq \Gamma(x)$ and lev $(s t) \sqsubseteq \Gamma(x)$. By the typing rules, it holds that $l e v(e) \sqsubseteq \Gamma(x)$ and $p c \sqsubseteq \Gamma(x)$. By these two facts, and having that $l e v(s t) \sqsubseteq p c$, it holds that $l e v(e) \sqsubseteq \Gamma(x)$ and $\operatorname{lev}(s t) \sqsubseteq \Gamma(x)$.

$\left.c_{1} ; c_{2}\right)$ Given a st such that $\operatorname{lev}(s t) \sqsubseteq p c$, we need to prove that exists $s t^{\prime}$ such that

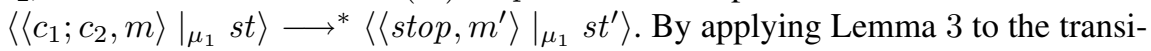
tion $\mu_{0}$ in the hypothesis, it holds that there exists $c^{*}, m^{\prime \prime}, s t^{*}$ such that $c^{\prime}=c^{*} ; c_{2}$ ) In this case, we have that

$$
\left\langle\left.\left\langle c_{1}, m\right\rangle\right|_{\mu_{0}} \epsilon\right\rangle \longrightarrow^{*}\left\langle\left.\left\langle c^{*}, m^{\prime}\right\rangle\right|_{\mu_{0}} s t^{*}\right\rangle
$$

We know that $s t^{*}=\epsilon$ from the definition of $\mu_{0}$. We apply IH on $p c \vdash c_{1}$ (obtaining from the typing rules) and (1), then we obtain that $\forall \operatorname{lev}\left(s t_{1}\right) \sqsubseteq$ $p c \cdot \exists \operatorname{lev}\left(s t_{1}^{\prime}\right) \cdot\left\langle\left.\left\langle c_{1}, m\right\rangle\right|_{\mu_{1}} s t_{1}\right\rangle \longrightarrow^{*}\left\langle\left.\left\langle c^{*}, m^{\prime}\right\rangle\right|_{\mu_{1}} s t_{1}^{\prime}\right\rangle$. Let's instantiate this formula by taking $s t_{1}=s t$. We then have that

$$
\left\langle\left.\left\langle c_{1}, m\right\rangle\right|_{\mu_{1}} s t\right\rangle \longrightarrow^{*}\left\langle\left.\left\langle c^{*}, m^{\prime}\right\rangle\right|_{\mu_{1}} s t_{1}^{\prime}\right\rangle
$$

By Lemma 2 applied to (2) and $c_{2}$, we obtain the transition $\left\langle\left.\left\langle c_{1} ; c_{2}, m\right\rangle\right|_{\mu_{1}} s t\right\rangle$ $\left.c^{\prime} \neq c^{*} ; c_{2}\right)$

$$
\begin{aligned}
& \left\langle\left.\left\langle c_{1}, m\right\rangle\right|_{\mu_{0}} \epsilon\right\rangle \longrightarrow^{*}\left\langle\left.\left\langle\text { stop, } m^{\prime \prime}\right\rangle\right|_{\mu_{0}} \epsilon\right\rangle \\
& \left\langle\left.\left\langle c_{2}, m^{\prime \prime}\right\rangle\right|_{\mu_{0}} \epsilon\right\rangle \longrightarrow^{*}\left\langle\left.\left\langle c^{\prime}, m^{\prime}\right\rangle\right|_{\mu_{0}} \epsilon\right\rangle
\end{aligned}
$$

We omit the proof when $\left\langle\left.\left\langle c_{2}, m^{\prime \prime}\right\rangle\right|_{\mu_{0}} \epsilon\right\rangle \longrightarrow_{0}\left\langle\left.\left\langle c^{\prime}, m^{\prime}\right\rangle\right|_{\mu_{0}} \epsilon\right\rangle$ since it follows as the previous case. We apply IH on $p c \vdash c_{1}$ (obtaining from the typing rules) and (3), then we obtain that $\forall \operatorname{lev}\left(s t_{1}\right) \sqsubseteq p c \cdot \exists \operatorname{lev}\left(s t_{1}^{\prime}\right) \cdot\left\langle\left.\left\langle c_{1}, m\right\rangle\right|_{\mu_{1}} s t_{1}\right\rangle \longrightarrow *$ $\left\langle\left.\left\langle s t o p, m^{\prime \prime}\right\rangle\right|_{\mu_{1}} s t_{1}^{\prime}\right\rangle$. Let's instantiate this formula by taking $s t_{1}=s t$. We then have that

$$
\left\langle\left.\left\langle c_{1}, m\right\rangle\right|_{\mu_{1}} s t\right\rangle \longrightarrow^{*}\left\langle\left.\left\langle s t o p, m^{\prime \prime}\right\rangle\right|_{\mu_{1}} s t_{1}^{\prime}\right\rangle
$$

From Lemma 1 , we have that $s t_{1}^{\prime}=s t$. Similarly, by instantiating the formula obtained by IH on $p c \vdash c_{2}$ and (4), we have that

$$
\left\langle\left.\left\langle c_{2}, m^{\prime \prime}\right\rangle\right|_{\mu_{1}} s t\right\rangle \longrightarrow{ }^{*}\left\langle\left.\left\langle c^{\prime}, m^{\prime}\right\rangle\right|_{\mu_{1}} s t_{2}^{\prime}\right\rangle
$$

By Lemma 2 applied to (5), we have $\left\langle\left.\left\langle c_{1} ; c_{2}, m\right\rangle\right|_{\mu_{1}} s t\right\rangle \longrightarrow^{*}\left\langle\left.\left\langle c_{2}, m^{\prime \prime}\right\rangle\right|_{\mu_{1}} s t\right\rangle$. The result follows from $(6)$. 
if $e$ then $c_{1}$ else $c_{2}$ ) Let's assume that $m(e) \neq 0$ (the proof follows the same structure when $m(e)=0$ ). We omit the proof when $\longrightarrow_{0}$ since it holds trivially. By semantics, we know that

$$
\begin{aligned}
& \left\langle\left.\left\langle\text { if } e \text { then } c_{1} \text { else } c_{2}, m\right\rangle\right|_{\mu_{0}} \epsilon\right\rangle \longrightarrow\left\langle\left.\left\langle c_{1} ; \text { end, } m\right\rangle\right|_{\mu_{0}} \epsilon\right\rangle \\
& \left\langle\left.\left\langle c_{1} ; \text { end, } m\right\rangle\right|_{\mu_{0}} \epsilon\right\rangle \longrightarrow{ }^{*}\left\langle\left.\left\langle c^{\prime}, m^{\prime}\right\rangle\right|_{\mu_{0}} \epsilon\right\rangle
\end{aligned}
$$

By definition of the monitor, we know that

$$
\left\langle\left.\left\langle\text { if } e \text { then } c_{1} \text { else } c_{2}, m\right\rangle\right|_{\mu_{1}} s t\right\rangle \longrightarrow\left\langle\left.\left\langle c_{1} ; \text { end, } m\right\rangle\right|_{\mu_{1}} l e v(e): s t\right\rangle
$$

If $\longrightarrow^{*}$ is $\longrightarrow_{0}$ in (8), the result follows from (9). Otherwise, by applying Lemma 3 on (8) and semantics, we have that there exists $m^{\prime \prime}, c^{*}$, and $s t^{*}$ such that $\left.c^{\prime}=c^{*} ; e n d\right)$ In this case, we have that

$$
\left\langle\left.\left\langle c_{1}, m\right\rangle\right|_{\mu_{0}} \epsilon\right\rangle \longrightarrow^{*}\left\langle\left.\left\langle c^{*}, m^{\prime}\right\rangle\right|_{\mu_{0}} s t^{*}\right\rangle
$$

We know that $s t^{*}=\epsilon$ from the definition of $\mu_{0}$. We apply IH on lev $(e) \sqcup p c \vdash$ $c_{1}$ (obtaining from the typing rules) and (10), then we obtain that $\forall \operatorname{lev}\left(s t_{1}\right) \sqsubseteq$ $\operatorname{lev}(e) \sqcup p c \cdot \exists \operatorname{lev}\left(s t_{1}^{\prime}\right) \cdot\left\langle\left.\left\langle c_{1}, m\right\rangle\right|_{\mu_{1}} s t_{1}\right\rangle \longrightarrow{ }^{*}\left\langle\left.\left\langle c^{*}, m^{\prime}\right\rangle\right|_{\mu_{1}} s t_{1}^{\prime}\right\rangle$. Let's instantiate this formula by taking $s t_{1}=\operatorname{lev}(e): s t$. We then have that

$$
\left\langle\left.\left\langle c_{1}, m\right\rangle\right|_{\mu_{1}} \operatorname{lev}(e): s t\right\rangle \longrightarrow^{*}\left\langle\left.\left\langle c^{*}, m^{\prime}\right\rangle\right|_{\mu_{1}} s t_{1}^{\prime}\right\rangle
$$

By Lemma 2 applied to (11) and end, we obtain $\left\langle\left.\left\langle c_{1} ;\right.\right.$ end, $\left.\left.m\right\rangle\right|_{\mu_{1}} \operatorname{lev}(e): s t\right\rangle$ $c^{\prime} \neq c^{*} ;$ end $)$

$$
\begin{aligned}
& \left\langle\left.\left\langle c_{1}, m\right\rangle\right|_{\mu_{0}} \epsilon\right\rangle \longrightarrow^{*}\left\langle\left.\left\langle\text { stop, } m^{\prime \prime}\right\rangle\right|_{\mu_{0}} \epsilon\right\rangle \\
& \left\langle\left.\left\langle\text { end, } m^{\prime \prime}\right\rangle\right|_{\mu_{0}} \epsilon\right\rangle \longrightarrow{ }^{*}\left\langle\left.\left\langle c^{\prime}, m^{\prime}\right\rangle\right|_{\mu_{0}} \epsilon\right\rangle
\end{aligned}
$$

By IH on lev(e) $\vdash p \vdash c_{1}$ (obtaining from the typing rules) and (12), we have that $\forall \operatorname{lev}\left(s t_{1}\right) \sqsubseteq \operatorname{lev}(e) \sqcup p c \cdot \exists \operatorname{lev}\left(s t_{1}^{\prime}\right) \cdot\left\langle\left\langle c_{1}, m\right\rangle \mid \mu_{\mu_{1}} s t_{1}\right\rangle \longrightarrow^{*}$ $\left\langle\left\langle s t o p, m^{\prime \prime}\right\rangle \mid \mu_{1} s t_{1}^{\prime}\right\rangle$. Let's instantiate this formula with $s t_{1}=\operatorname{lev}(e): s t$. We then have that

$$
\left\langle\left.\left\langle c_{1}, m\right\rangle\right|_{\mu_{1}} \operatorname{lev}(e): s t\right\rangle \longrightarrow^{*}\left\langle\left.\left\langle s t o p, m^{\prime \prime}\right\rangle\right|_{\mu_{1}} s t_{1}^{\prime}\right\rangle
$$

At this point, we do not know the shape of $s t_{1}^{\prime}$, but we can deduced it by applying the Lemma 1 to it: $s t_{1}^{\prime}=\operatorname{lev}(e): s t$. Then, by Lemma 2 on (14) and semantics for $e n d$, we have that

$$
\left\langle\left.\left\langle c_{1} ; e n d, m\right\rangle\right|_{\mu_{1}} \operatorname{lev}(e): s t\right\rangle \longrightarrow^{*}\left\langle\left.\left\langle e n d, m^{\prime \prime}\right\rangle\right|_{\mu_{1}} \operatorname{lev}(e): s t\right\rangle
$$

In the case that $\longrightarrow^{*}$ is $\longrightarrow_{0}$ in (13), the result holds from (9) and (15). Otherwise, from semantics rules in (13), we know that $c^{\prime}=$ stop and $m^{\prime}=m^{\prime \prime}$. By monitor semantics, we know that

$$
\left\langle\left.\left\langle e n d, m^{\prime \prime}\right\rangle\right|_{\mu_{1}} \operatorname{lev}(e): s t\right\rangle \longrightarrow\left\langle\left.\left\langle s t o p, m^{\prime \prime}\right\rangle\right|_{\mu_{1}} s t\right\rangle
$$

The result then follows from (9), (15), and (16). 
while $e$ do $c$ ) Similar to the previous case.

We can then prove the first theorem.

Theorem 1. If $p c \vdash c$ and $\left\langle\langle c, m\rangle \mid \mu_{\mu_{0}} \epsilon\right\rangle \longrightarrow^{*}\left\langle\left\langle\right.\right.$ stop, $\left.\left.m^{\prime}\right\rangle \mid \mu_{\mu_{0}} \epsilon\right\rangle$, then $\left\langle\left.\langle c, m\rangle\right|_{\mu_{1}} \epsilon\right\rangle \longrightarrow^{*}$ $\left\langle\left.\left\langle s t o p, m^{\prime}\right\rangle\right|_{\mu_{1}} s t^{\prime}\right\rangle$.

Proof. By Lemma 4, we obtain that $\forall \operatorname{lev}(s t) \sqsubseteq p c \cdot \exists \operatorname{lev}\left(s t^{\prime}\right) \cdot\left\langle\left.\langle c, m\rangle\right|_{\mu_{1}} s t\right\rangle \longrightarrow *$ $\left\langle\left\langle s t o p, m^{\prime}\right\rangle \mid \mu_{1} s t^{\prime}\right\rangle$. The result follows by instantiating the formula with $s t=\epsilon$ since $\operatorname{lev}(\epsilon)=L$.

To prove Theorem 2, we firstly prove that, for terminating programs, there is an isomorphism between the command semantics and executions under $\mu_{0}$.

Lemma 5. Given command $c$ that contains no end instructions, $\langle c, m\rangle \longrightarrow^{*}\left\langle\right.$ stop, $\left.m^{\prime}\right\rangle$ $\Leftrightarrow\left\langle\left.\langle c, m\rangle\right|_{\mu_{0}} \epsilon\right\rangle \longrightarrow^{*}\left\langle\left.\left\langle s t o p, m^{\prime}\right\rangle\right|_{\mu_{0}} \epsilon\right\rangle$.

Proof. Both directions of the implication are proved by a simple induction on $\longrightarrow$.

Now, we are in conditions to prove the mentioned Theorem.

Theorem 2. If $p c \vdash c$, then for all $m_{1}$ and $m_{2}$, where $m_{1}={ }_{L} m_{2}$, whenever we have $\left\langle\left.\left\langle c, m_{1}\right\rangle\right|_{\mu_{0}} \epsilon\right\rangle \longrightarrow^{*}\left\langle\left.\left\langle\right.\right.$ stop, $\left.\left.m_{1}^{\prime}\right\rangle\right|_{\mu_{0}} \epsilon\right\rangle$ and $\left\langle\left.\left\langle c, m_{2}\right\rangle\right|_{\mu_{0}} \epsilon\right\rangle \longrightarrow^{*}\left\langle\left.\left\langle s t o p, m_{2}^{\prime}\right\rangle\right|_{\mu_{0}} \epsilon\right\rangle$, then $m_{1}^{\prime}={ }_{L} m_{2}^{\prime}$.

Proof. By Lemma 5, we have that $\left\langle c, m_{1}\right\rangle \longrightarrow^{*}\left\langle\right.$ stop, $\left.m_{1}^{\prime}\right\rangle$ and $\left\langle c, m_{2}\right\rangle \longrightarrow^{*}\left\langle\right.$ stop, $\left.m_{2}^{\prime}\right\rangle$. The result follows by applying the soundness theorem from [27] to $p c \vdash c,\left\langle c, m_{1}\right\rangle \longrightarrow$ * $\left\langle\right.$ stop, $\left.m_{1}^{\prime}\right\rangle$, and $\left\langle c, m_{2}\right\rangle \longrightarrow^{*}\left\langle\right.$ stop,$\left.m_{2}^{\prime}\right\rangle$.

We need two auxiliary lemmas in order to prove Theorem 3. They express that public variables cannot be affected when the security level of the monitor's stack is $H$.

Lemma 6. If $c$ contains no end instructions, lev $(s t)=H$, and $\left\langle\langle c, m\rangle \mid \mu_{\mu_{1}} s t\right\rangle \longrightarrow$ * $\left\langle\left\langle\right.\right.$ stop, $\left.\left.m^{\prime}\right\rangle\right| \mu_{\mu_{1}}$ st $\rangle$, then $m={ }_{L} m^{\prime}$.

Proof. By induction on $\longrightarrow$.

Lemma 7. If $c$ contains no end instructions, and $\langle\langle$ while $e$ do $c, m\rangle| \mu_{1}$ st $\rangle \longrightarrow^{*}$ $\left\langle\left.\left\langle\right.\right.$ stop, $\left.m^{\prime}\right\rangle\right|_{\mu_{1}}$ st $\rangle$, then $m={ }_{L} m^{\prime}$.

Proof. By performing one small-step in the semantics and then applying Lemma 6.

The next lemma is a generalization of Theorem 3 .

Lemma 8. For all $m_{1}$ and $m_{2}$, where $m_{1}={ }_{L} m_{2}$, whenever $c$ contains no end commands and $\left\langle\left.\left\langle c, m_{1}\right\rangle\right|_{\mu_{1}} s t\right\rangle \longrightarrow^{*}\left\langle\left.\left\langle s t o p, m_{1}^{\prime}\right\rangle\right|_{\mu_{1}}\right.$ st $\left.t_{1}^{\prime}\right\rangle$ and $\left\langle\left.\left\langle c, m_{2}\right\rangle\right|_{\mu_{1}} s t\right\rangle \longrightarrow^{*}$ $\left\langle\left\langle s t o p, m_{2}^{\prime}\right\rangle \mid{ }_{\mu_{1}} s t_{2}^{\prime}\right\rangle$, then $m_{1}^{\prime}=_{L} m_{2}^{\prime}$.

Proof. By induction on $\longrightarrow$ *. We list the most interesting cases. 
$c_{1} ; c_{2}$ ) By applying Lemma 3 to our hypothesis, we obtain that there exists $m_{1}^{\prime \prime}$ and $m_{2}^{\prime \prime}$ such that

$$
\begin{aligned}
& \left\langle\left.\left\langle c_{1}, m_{1}\right\rangle\right|_{\mu_{1}} s t\right\rangle \longrightarrow^{*}\left\langle\left.\left\langle s t o p, m_{1}^{\prime \prime}\right\rangle\right|_{\mu_{1}} s t\right\rangle \\
& \left\langle\left.\left\langle c_{2}, m_{1}^{\prime \prime}\right\rangle\right|_{\mu_{1}} s t\right\rangle \longrightarrow^{*}\left\langle\left.\left\langle s t o p, m_{1}^{\prime}\right\rangle\right|_{\mu_{1}} s t_{1}^{\prime}\right\rangle \\
& \left\langle\left.\left\langle c_{1}, m_{2}\right\rangle\right|_{\mu_{1}} s t\right\rangle \longrightarrow^{*}\left\langle\left.\left\langle s t o p, m_{2}^{\prime \prime}\right\rangle\right|_{\mu_{1}} s t\right\rangle \\
& \left\langle\left.\left\langle c_{2}, m_{2}^{\prime \prime}\right\rangle\right|_{\mu_{1}} s t\right\rangle \longrightarrow^{*}\left\langle\left.\left\langle s t o p, m_{2}^{\prime}\right\rangle\right|_{\mu_{1}} s t_{2}^{\prime}\right\rangle
\end{aligned}
$$

By IH on $m_{1}={ }_{L} m_{2},(17)$ and (19), we obtain that $m_{1}^{\prime \prime}={ }_{L} m_{2}^{\prime \prime}$. By IH on $m_{1}^{\prime \prime}={ }_{L}$ $m_{2}^{\prime \prime}$, (18), and (20), we have that $m_{1}^{\prime}={ }_{L} m_{2}^{\prime}$ as expected.

if $e$ then $c_{1}$ else $c_{2}$ ) We consider the case when $\operatorname{lev}(e)=H$ and that $m_{1}(e) \neq$ $m_{2}(e)$. Otherwise, the proof follows by simply applying $\mathrm{IH}$ and Lemmas 2 and 3. We assume, without loosing generality, that $m_{1}(e) \neq 0$. Consequently, by semantics, we have that

$$
\begin{aligned}
& \left\langle\left.\left\langle\text { if } e \text { then } c_{1} \text { else } c_{2}, m_{1}\right\rangle\right|_{\mu_{1}} s t\right\rangle \longrightarrow\left\langle\left.\left\langle c_{1} ; \text { end, } m_{1}\right\rangle\right|_{\mu_{1}} \operatorname{lev}(e): s t\right\rangle(21) \\
& \left\langle\left.\left\langle c_{1} ; \text { end, } m_{1}\right\rangle\right|_{\mu_{1}} \operatorname{lev}(e): s t\right\rangle \longrightarrow^{*}\left\langle\left.\left\langle s t o p, m_{1}^{\prime}\right\rangle\right|_{\mu_{1}} s t_{1}^{\prime}\right\rangle \\
& \left\langle\left.\left\langle\text { if } e \text { then } c_{1} \text { else } c_{2}, m_{2}\right\rangle\right|_{\mu_{1}} s t\right\rangle \longrightarrow\left\langle\left.\left\langle c_{2} ; \text { end, } m_{2}\right\rangle\right|_{\mu_{1}} \operatorname{lev}(e): s t\right\rangle \\
& \left\langle\left.\left\langle c_{2} ; \text { end }, m_{2}\right\rangle\right|_{\mu_{1}} \operatorname{lev}(e): s t\right\rangle \longrightarrow^{*}\left\langle\left\langle s t o p, m_{2}^{\prime}\right\rangle \mid \mu_{\mu_{1}} s t_{2}^{\prime}\right\rangle
\end{aligned}
$$

By applying Lemma 3 on (22) and (24), we have that there exists $m_{1}^{\prime \prime}$ and $m_{2}^{\prime \prime}$ such that

$$
\begin{aligned}
& \left\langle\left\langle c_{1}, m_{1}\right\rangle \mid{ }_{\mu_{1}} \operatorname{lev}(e): s t\right\rangle \longrightarrow^{*}\left\langle\left.\left\langle s t o p, m_{1}^{\prime \prime}\right\rangle\right|_{\mu_{1}} \operatorname{lev}(e): s t\right\rangle \\
& \left\langle\left.\left\langle e n d, m_{1}^{\prime \prime}\right\rangle\right|_{\mu_{1}} \operatorname{lev}(e): s t\right\rangle \longrightarrow^{*}\left\langle\left.\left\langle s t o p, m_{1}^{\prime}\right\rangle\right|_{\mu_{1}} s t_{1}^{\prime}\right\rangle \\
& \left\langle\left.\left\langle c_{2}, m_{2}\right\rangle\right|_{\mu_{1}} \operatorname{lev}(e): s t\right\rangle \longrightarrow^{*}\left\langle\left.\left\langle s t o p, m_{2}^{\prime \prime}\right\rangle\right|_{\mu_{1}} \operatorname{lev}(e): s t\right\rangle \\
& \left\langle\left\langle e n d, m_{2}^{\prime \prime}\right\rangle \mid \mu_{\mu_{1}} l e v(e): s t\right\rangle \longrightarrow^{*}\left\langle\left\langle s t o p, m_{2}^{\prime}\right\rangle \mid \mu_{\mu_{1}} s t_{2}^{\prime}\right\rangle
\end{aligned}
$$

By applying Lemma 6 on (25) and (27), we have that $m_{1}^{\prime \prime}=_{L} m_{1}={ }_{L} m_{2}={ }_{L} m_{2}^{\prime \prime}$. By semantics, (26), and (28), we have that $m_{1}^{\prime}=m_{1}^{\prime \prime}$ and $m_{2}^{\prime}=m_{2}^{\prime \prime}$. Consequently, we have that $m_{1}^{\prime}={ }_{L} m_{2}^{\prime}$ as expected.

while $e$ do $c$ ) The proof proceeds similarly as the previous case but also applying Lemma 7 when needed.

We prove our last last theorem as follows.

Theorem 3. For all $m_{1}$ and $m_{2}$, where $m_{1}={ }_{L} m_{2}$, whenever c contains no end commands and $\left\langle\left.\left\langle c, m_{1}\right\rangle\right|_{\mu_{1}} \epsilon\right\rangle \longrightarrow{ }^{*}\left\langle\left.\left\langle s t o p, m_{1}^{\prime}\right\rangle\right|_{\mu_{1}}\right.$ st $\left.t_{1}^{\prime}\right\rangle$ and $\left\langle\left.\left\langle c, m_{2}\right\rangle\right|_{\mu_{1}} \epsilon\right\rangle \longrightarrow^{*}$ $\left\langle\left.\left\langle\right.\right.$ stop, $\left.\left.m_{2}^{\prime}\right\rangle\right|_{\mu_{1}} s t_{2}^{\prime}\right\rangle$, then $m_{1}^{\prime}={ }_{L} m_{2}^{\prime}$.

Proof. By applying Lemma 8 with $s t=\epsilon$. 